www.nature.com/pcan

\title{
EDITORIAL
}

\section{Report from Durham}

Prostate Cancer and Prostatic Diseases (2008) 11, 1; doi:10.1038/pcan.2008.8

Welcome to the first issue of 2008! We are very excited about this issue and the continued progress in research on prostate cancer and benign prostatic conditions. On this side of the pond, we are in the heat of the presidential primary season. As the United States will be changing leadership, this is an important issue related to prostate disease. All North American readers are aware of budget cuts over the past few years at the National Institutes of Health $(\mathrm{NIH})$, the United States leading biomedical research funding agency. Those of us in the field are watching closely, as election results may have a significant impact on future research funding. Furthermore, the health coverage challenge in the United States is substantial and we may be facing a 'perfect storm' as the population ages, there is a growing shortage of doctors and the proportion of uninsured citizens grows. The impact of these changes on prostate cancer and prostatic diseases is speculative, but portends interesting times ahead.

We begin this issue with four outstanding review articles. Von Low and colleagues offer a timely review on soy isoflavones in prostate cancer prevention and treatment. Like so many of the complementary and alternative medicine (CAM) strategies that many of our patients are interested in, the data on soy are intriguing but not definitive as the review expertly points out. The second review by my Duke University colleague, Dr Tom Polascik, evaluates bone health strategies in the setting of androgen deprivation for prostate cancer and the role of bisphosphonates. It is especially important for elderly men receiving longer-term hormonal therapy to be screened for osteoporosis and to be treated with vitamin D, calcium and a bisphosphonate if they have osteopenia/osteoporosis or are at substantial risk of developing these lifethreatening conditions. The third review by Godoy and Taneja is a comprehensive, excellent overview of highgrade prostatic intraepithelial neoplasia (HGPIN). Despite better identification of coexistent cancer due to extended prostate biopsy strategies, the diagnosis of HGPIN generally warrants active surveillance and periodic repeat biopsy depending on clinical suspicion. The final review article by Miki and Rhim is a critically important overview of prostate cancer cell lines, cell culture and prostate stem cells. Dr Rhim is a world authority on prostate cell cultures and a loyal supporter of this journal, and we sincerely appreciate his contributions.

We next feature six clinical science original contributions led off by results of a controlled randomized clinical trial (RCT). Ng et al. report on a multicenter RCT of prostatic cryotherapy (CRYO) versus external beam radiotherapy (EBRT) in clinical T3 disease. Both arms also received 6 months of hormonal therapy. While the trial accrued only 64 out of the planned 150 men, the results are provocative. The 4-year biochemical disease-free survival was $47 \%$ for EBRT and only $13 \%$ for CRYO and the author's state that
CRYO is a suboptimal therapy for T3 disease. These results and conclusions are sure to raise discussion among readers! Speaking of controversy, Fleshner and colleagues contribute a provocative article arguing that primary hormonal therapy use in non-metastatic prostate cancer can be considered curative and not palliative in many cases. Certainly, this piece will also engender debate in the pro and con hormonal camps throughout the world.

In the third original article, Tamin et al. examine tricyclic antidepressants (TCAs) and risk of prostate cancer. To our knowledge, this is the first paper of this type on this topic. The authors found a small dose-dependent increased risk of prostate cancer with TCAs, which now must be confirmed or refuted by additional work. Ambrosini and associates next examine fruit, vegetable and vitamin A intake and the risk of prostate cancer. Again, just as with CAMs, there is tremendous interest in this topic among our patients and their families. Unfortunately, despite what common sense and common perception would suggest, this study failed to show risk reduction and further study is needed. Wright and colleagues, using the CaPSURE database, look at the timely topic of radical prostatectomy ( $R P)$ outcomes in young men $(<55$ years) finding less impact of the surgery on sexual and urinary outcomes compared to older men. As an experienced radical prostatectomist myself, these results are in line with our current work from the Duke Prostate Center. Whether performed by robotic or minimally invasive open technique, today's RP performed in experienced hands is a far cry from the surgery of old! Our current message to young men is that 'life goes on' and in a significantly unaltered state for the vast majority. On the topic of RP, Manoharan et al. report on $544 \mathrm{RP}$ cases performed via a Pfannenstiel incision. This paper is a very good example of surgical excellence due to the skill and dedication of an experienced radical prostatectomist.

To round out the issue, we have three basic/translational original articles. Nanus et al. demonstrate eloquently that neutral endopeptidase is involved in prostate tumorigenesis and may be a future therapeutic target. Drewa et al. provide further support that doxazocin, commonly used to treat the symptoms of $\mathrm{BPH}$, causes apoptosis in prostate cells due to receptor cross talk rather than one receptor pathway. Finally, Medeiros and associates studied 904 men showing that FAS polymorphisms may play a role in the risk of prostate cancer susceptibility.

Two interesting case reports close out this issue. Gacci et al. nicely demonstrate that brachytherapy seed migration after proper initial placement can sometimes be responsible for therapy failure. Ferro et al. report on the rare novel case of chyluria associated with advanced prostate cancer.

Thank you for your continued support of Prostate Cancer and Prostatic Diseases. 